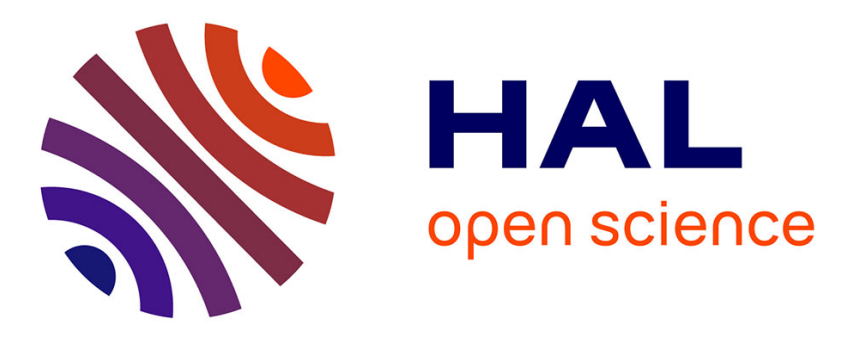

\title{
Les divergences au sein de la composante technico-scientifique du nouveau système sectoriel d'innovation de la chimie doublement verte
}

Estelle Garnier, Romain Debref

\section{- To cite this version:}

Estelle Garnier, Romain Debref. Les divergences au sein de la composante technico-scientifique du nouveau système sectoriel d'innovation de la chimie doublement verte. Innovations - Revue d'économie et de management de l'innovation, 2014, 43 (1), pp.39. 10.3917/inno.043.0039 . hal-02047208

\section{HAL Id: hal-02047208 \\ https://hal.univ-reims.fr/hal-02047208}

Submitted on 1 Jul 2020

HAL is a multi-disciplinary open access archive for the deposit and dissemination of scientific research documents, whether they are published or not. The documents may come from teaching and research institutions in France or abroad, or from public or private research centers.
L'archive ouverte pluridisciplinaire $\mathbf{H A L}$, est destinée au dépôt et à la diffusion de documents scientifiques de niveau recherche, publiés ou non, émanant des établissements d'enseignement et de recherche français ou étrangers, des laboratoires publics ou privés. 


\section{LES DIVERGENCES AU SEIN DE LA COMPOSANTE TECHNICO- SCIENTIFIQUE DU NOUVEAU SYSTĖME SECTORIEL D'INNOVATION DE LA CHIMIE DOUBLEMENT VERTE} Estelle Garnier, Romain Debref

De Boeck Supérieur | «Innovations »

2014/1 n 43 | pages 39 à 59

ISSN 1267-4982

ISBN 9782804187644

Article disponible en ligne à l'adresse :

https://www.cairn.info/revue-innovations-2014-1-page-39.htm

\section{Pour citer cet article :}

Estelle Garnier, Romain Debref« Les divergences au sein de la composante technicoscientifique du nouveau système sectoriel d'innovation de la chimie doublement verte », Innovations 2014/1 ( $\left.\mathrm{n}^{\circ} 43\right)$, p. 39-59.

DOI 10.3917/inno.043.0039

Distribution électronique Cairn.info pour De Boeck Supérieur.

(C) De Boeck Supérieur. Tous droits réservés pour tous pays.

La reproduction ou représentation de cet article, notamment par photocopie, n'est autorisée que dans les limites des conditions générales d'utilisation du site ou, le cas échéant, des conditions générales de la licence souscrite par votre établissement. Toute autre reproduction ou représentation, en tout ou partie, sous quelque forme et de quelque manière que ce soit, est interdite sauf accord préalable et écrit de l'éditeur, en dehors des cas prévus par la législation en vigueur en France. Il est précisé que son stockage dans une base de données est également interdit. 


\title{
LES DIVERGENCES AU SEIN DE LA COMPOSANTE TECHNICO-SCIENTIFIQUE DU NOUVEAU SYSTĖME SECTORIEL D'INNOVATION DE LA CHIMIE DOUBLEMENT VERTE ${ }^{1}$
}

\author{
Estelle GARNIER \\ REGARDS (EA 6292) \\ Université de Reims Champagne-Ardenne \\ estelle.garnier@utt.fr \\ Roman DEBREF? \\ REGARDS (EA 6292) \\ Université de Reims Champagne-Ardenne \\ romain.debref@univ-reims.fr
}

L'idée de développer une chimie doublement verte ( C2V » dans le corps de texte) nait à la convergence de dynamiques structurelles venant impacter les secteurs d'activité traditionnels que sont l'agriculture et la chimie. Ces mouvements sont de trois ordres. Il s'agit premièrement des excédents agricoles et de la recherche de nouveaux débouchés non alimentaires dans la sphère agricole. Il s'agit ensuite de la survenue du peak oil et de la recherche de substituts aux produits issus du pétrole dans le secteur de la chimie. Il s'agit enfin de la montée en puissance de la thématique du développement durable dans la société dans son ensemble et de sa traduction en termes de Green Chemistry dans le domaine de la chimie. Si le concept de chimie doublement verte se présente comme une solution à ces trois problématiques

1. Pour ce papier, les auteurs ont reçu le prix « Jeune Chercheur» du Réseau de Recherche sur l'Innovation lors de son école d'été : «Les nouvelles dimensions des systèmes sectoriels de l'innovation », Montpellier, 29 août - 1er septembre 2012.

2. Ce papier s'est nourri des réflexions menées conjointement avec les autres membres de l'ANR APERC2V (Nieddu, Kurek, Bliard et Vivien) que nous tenons à remercier ici. Nous tenons également à remercier les évaluateurs anonymes pour leurs remarques et leurs contributions à la qualité de ce travail. 
structurelles : elle permet de se passer des ressources fossiles en valorisant, par des process durables, les ressources agricoles ; l'étude plus fine des effets de ces trois dynamiques structurelles montre que ceux-ci ne sont pas directs ou homogènes. En premier lieu, si chacun des mouvements structuraux créer des «fenêtres d'opportunités » (Nill, 2003) pour aller vers une chimie doublement verte, ils orientent aussi les acteurs de la recherche vers des voies de développement qui peuvent être parfois complémentaires, parfois indépendantes, mais aussi parfois contradictoires entre elles. En second lieu, au-delà de l'impératif de valoriser du végétal de manière durable, l'idée de développer une chimie biosourcée durable est tellement ouverte qu'elle laisse la possibilité aux acteurs de se positionner par rapport à l'un ou l'autre de ces mouvements tout en le faisant de manière différenciée ${ }^{3}$. In fine, si la chimie doublement verte en tant que concept se pose comme la solution unique à plusieurs problèmes structurels, la façon dont le secteur chimie doublement verte se met en place est difficile à appréhender.

Le courant de sustainability transition management nous fournit un cadre d'analyse des évolutions dans les secteurs de la chimie et de l'agriculture. Celui-ci se présente dans un ouvrage de synthèse récent (Grin et al., 2010), comme une construction qui vise à unifier les apports respectifs des Science $\mathcal{G}$ Technology Studies, de l'économie évolutionniste des systèmes sectoriels d'innovation et de production, et d'une sociologie s'inspirant de la théorie de la structuration de Giddens, dans une théorie générale de la transition d'un régime socio-technique à un autre, et qui a pour objectif de proposer un « paradigm for sustainable innovation policies» (Nill, Kemp, 2009, p. 677).

Nous pouvons comprendre cette transition en combinant deux approches complémentaires qui ont fait leur preuve. La première repose sur la théorie du transition to sustainable developement (Kemp, 2001 ; Kemp et al., 1998 ; Geels, 2002, 2005a, 2005b, 2010, 2011 ; Green, Rotmans, Schot, 2010), qui nous permet de saisir ce qui influence les déterminants de l'évolution d'un secteur à différents niveaux. Cette perspective multi-niveau ${ }^{4}$ qui fait la force de ces travaux intègre un caractère continu des changements, et nous invitent ainsi à appréhender l'émergence du secteur de la C2V à partir de dynamiques de moyen/long terme. Geels et ses collègues hollandais (Green, Rotmans, Schot, 2010), qui travaillent sur la transition vers des régimes sociotechniques durables, présentent les conditions d'apparition et

3. Par exemple, si la politique de jachères industrielles mise en œuvre pour résorber les excédents a conduit à orienter les dispositifs institutionnels et les recherches vers le développement des biocarburants, au travers de la stratégie «valorisation de la plante entière " le monde de l'agriculture a aussi exprimé des attentes vers des développements en chimie.

4. Plus connu sous le nom de Multilevel Perspective (MLP). 
de stabilisation de nouveaux secteurs d'activité. Ces phénomènes se réalisent à travers une dynamique en deux temps. Il y a d'abord une exploration des différentes voies de développement possible, puis une exploitation des voies qui s'avèrent être les plus pertinentes. L'exploration est un mouvement qui se met en place au niveau micro-économique dans des niches de spécialisation. La pertinence de ces niches (et donc leur exploitation) dépend, quant à elle, de mouvements au niveau intermédiaire et macro-économique. C'est-à-dire qu'elle est fonction 1) des régimes technico-économiques existants et 2) des évolutions du paysage globale. L'ensemble de ces influences parviendrait à expliquer la convergence vers un dominant design modifiant le(s) régime(s) sociotechnique(s) en place, voire même au-delà (cf. figure 1).

Figure 1 - La transition technologique dans une perspective dynamique

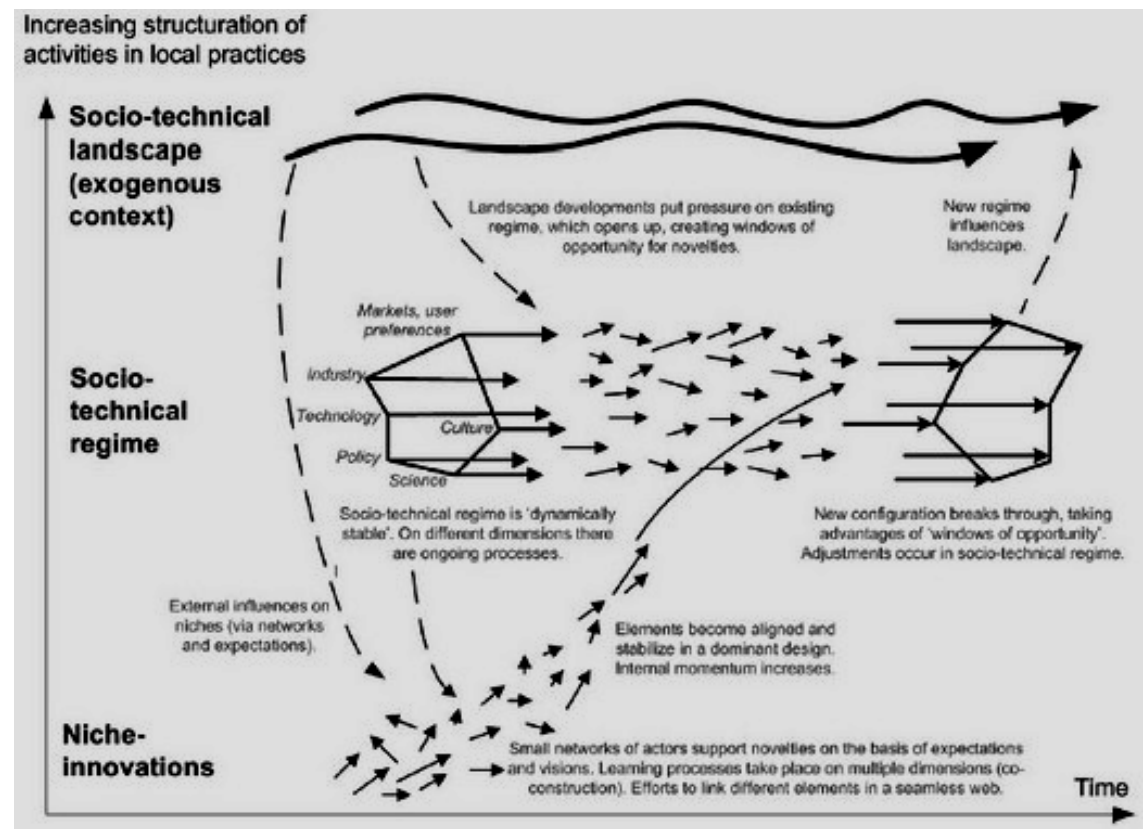

Source: Geels (2002)

Bien que cette approche soit indispensable pour appréhender la complexité de la transition, nous pensons que l'approche par les Systèmes Sectoriels d'Innovation et de Production (SSIP) (Malerba, 2002, 2004 ; Oltra, Saint Jean, 2007, 2009, 2010, 2012) est tout aussi importante, car les systèmes sectoriels évoluent et s'adaptent aux différentes influences 
multidimensionnelles. Malerba identifie cinq éléments principaux ${ }^{5}$ qui caractérisent les systèmes sectoriels. Il s'agit 1) des connaissances et des processus d'apprentissage, 2 ) des technologies de base, des intrants et de la demande, 3) des types et structures d'interactions entre un ensemble hétérogène d'entreprises et d'autres organisations, 4) des institutions et 5) des processus de génération de variété et de sélection (2002, p. 251). Oltra et Saint Jean qui travaillent plus spécifiquement sur les innovations environnementales précisent que les tendances sectorielles d'innovations environnementales sont le fruit de compromis technologiques visant à combiner les objectifs réglementaires et les performances environnementales avec les objectifs de productivité et de compétitivité des firmes $(2009$, p. 2). Leur propos souligne aussi l'existence de convergence de même ampleur. Finalement, on retiendra de cette combinaison « composite » un cadre d'analyse pour le «Système Sectoriel d'Innovation de la Chimie Doublement Verte » ( SSI $\mathrm{C} 2 \mathrm{~V}$ » dans le corps de texte) nous permettant de constater, ou non, des convergences, et dans tous les cas les expliquer.

Afin de préciser la manière dont le Système Sectoriel d'Innovation de la chimie doublement verte se met en place, ces deux éléments, nous invitent à nous interroger sur la dynamique d'évolution dans le temps de chacune des différentes composantes. Nous proposons de nous livrer à cet exercice pour une composante spécifique du Système Sectoriel d'Innovation de la Chimie Doublement Verte : celle des connaissances de base que nous avons renommées « composante technico-scientifique ${ }^{6}$. Nous la définissons comme l'ensemble des connaissances produites par la sphère scientifique et mobilisée dans les activités industrielles des acteurs à la pointe de l'innovation. C'est donc dans un tel contexte, que nous allons chercher s'il y a une convergence ou une divergence des dynamiques de cette composante qui orienterait de fait le Système Sectoriel d'Innovation de la Chimie Doublement Verte vers une voie technologique dominante unique, ou bien, vers une diversité de voies maintenue dans le temps.

Pour apporter une réponse à cette question, nous avons étudié cette dynamique dans une perspective multi-niveaux, c'est-à-dire à deux endroits différents du secteur. D'abord au niveau de la chimie académique. Comme un même produit fini peut être obtenu à partir de différentes matières premières

5. Qualifiés de building blocks (Malerba, 2002, p. 251).

6. Nous n'avons pas souhaité ici reprendre la terminologie « connaissances de base » proposée par Malerba pour la composante que nous avons étudiée et y avons préféré le terme « technico-scientifique » car notre position analytique est dynamique. Le choix de cette terminologie s'explique par le fait que, certes, nous traitons de la science mais dans une optique de mobilisation de celle-ci dans des voies techniques. 
associées à différents process chimiques et que dans le même temps on a un contexte de ressources limitées, la science chimique se doit de limiter les voies de recherche qu'elle investit. Aussi il est important d'identifier à ce niveau si on a effectivement un nombre de solutions scientifiques (ellesmêmes bases des voies technologiques) limité qui est investi. Ensuite au niveau des développements industriels afin de voir si le mouvement de réduction des voies technologiques qui est envisagé par le courant sustainable transition management se met bien à l'œuvre, engendrant par la même une réduction des bases scientifiques mobilisées.

Nous avons repris les résultats des travaux empiriques menés dans deux thèses (Garnier, 2012 ; Debref, en cours) et d'un projet ARN (AEPRC2V) s'appuyant sur différentes sources, dont principalement 1) le dépouillement des revues de référence dans les différentes sciences nécessaires au développement du concept de chimie verte ; 2 ) une analyse des textes de projets de recherche liés à l'usage de la biomasse ; 3) une veille sur les sites d'entreprises particulièrement impliquées dans le secteur ; 4) des entrevues avec les acteurs du secteur. Et nous avons mis en forme ces derniers au travers d'une approche narrative (Nieddu et al., 2010).

De cette manière, la première partie $(1)$ de notre travail nous permettra de distinguer deux étapes dans la représentation et le développement de la $\mathrm{C} 2 \mathrm{~V}$ et de souligner des divergences des représentations relatives à la mise en œuvre la $\mathrm{C} 2 \mathrm{~V}$ au niveau de la sphère académique. Dans notre deuxième partie (2), où nous traiterons du niveau des développements et des applications industriels dans le secteur des revêtements de sol résilients, le travail mené confirmera la coexistence de voies de développement relevant de la Green Chemistry avec des voies relevant de la «Chimie doublement verte ».

\section{LA DYNAMIQUE DE LA COMPOSANTE TECHNICO-SCIENTIFIQUE DU SYSTĖME SECTORIEL D'INNOVATION DE LA CHIMIE DOUBLEMENT VERTE AU NIVEAU ACADÉMIQUE}

Dans la mesure où elle constitue la principale force de production des connaissances de base à partir desquelles les recompositions des relations et des frontières intersectorielles vont pouvoir se réaliser (Nieddu et al., 2010), la science est un élément central dans la stratégie des acteurs économiques cherchant à assurer leur reproduction dans le temps dans un contexte de changement à venir. Il est donc nécessaire d'observer la manière dont la science influence la composante technico-scientifique des SSI et se diffuse dans le secteur de la C2V. 


\section{L'absence d'une voie scientifique dominante pour la chimie verte du végétal à partir la chimie durable et sa déclinaison en termes de Green Chemistry}

\section{De l'émergence à la dominance du concept de Green Chemistry}

À notre sens, l'histoire du développement de la chimie durable commence en 1990 et de façon prononcée aux États-Unis (Linthorst, 2009 ; Garnier, 2012). À cette époque, face aux problèmes environnementaux, le Ministère Américain de l'Environnement qui considère qu'il y a des possibilités significatives pour l'industrie de réduire ou d'empêcher la pollution à la source ratifie le Pollution Prevention Act. De par sa place dans l'économie et ses relations aux autres secteurs d'activités, le secteur de la chimie devient le premier secteur impacté par cette politique de prévention. L'époque voit alors fleurir un nombre important d'appellations et de concepts scientifiques renvoyant à l'idée d'une chimie durable : environmental chemistry, chimie soutenable, clean chemistry, begnin chemistry (Woodhouse, Breyman, 2005), chimie douce (Livage, 2009).

On peut considérer que le concept et l'appellation de Green Chemistry vont s'imposer dans le monde de la recherche en chimie en 1998 avec deux évènements importants. Le premier est la publication de Green Chemistry: Theory and Practice par Anastas et Warner. Celle-ci va proposer la définition la plus reprise aujourd'hui de la Green Chemistry : « la conception, le développement et l'élaboration des produits et procédés chimiques pour réduire ou éliminer l'usage et la génération des substances dangereuses pour la santé et l'environnement " (Anastas, Warner, 1998, notre traduction). Elle va également comporter l'énoncé des 12 principes (figure 2) qui, par le succès de leur diffusion, vont marquer définitivement le paysage scientifique. Par ailleurs, durant cette même année, le réseau anglais de l'université d'York va, à partir d'articles abondamment cités dans la communauté scientifique (Clark, 1998 (a), (b), 1999) et comportant le mot clé Green Chemistry, considérer que la situation est assez mûre pour créer l'année suivante une nouvelle revue : Green Chemistry.

\section{La Green Chemistry, un concept reposant sur des priorités et des compromis}

Le succès du concept dans la communauté des chimistes académiques au niveau international va s'expliquer de deux manières. D'abord, par l'importance des États-Unis dans le monde de la recherche en chimie. Ensuite, par sa formulation même. Les 12 principes qui constituent la Green Chemistry (figure 2) visent en effet à intégrer les modes de raisonnement qui se veulent fondateurs d'une nouvelle philosophie de la chimie pour le $21^{\mathrm{e}}$ siècle (Clark, Macquarrie, 2002, p. 2). Mais, la Green Chemistry, telle qu'elle est pensée 
par Anastas et Warner en 1998, se présente aussi comme une feuille de route reposant sur des principes à respecter «autant que faire se peut » pour aller vers une chimie durable (Anastas, Warner 1998 ; Lancaster 2002). Ainsi, le monde académique dispose d'un outil qui est à la fois marqué d'une symbolique très forte et qui est très malléable.

\section{Figure 2 - Les 12 principes de la Green Chemistry}

1. La prévention de la pollution à la source en évitant la production de résidus.

2. L'économie d'atomes et d'étapes [...] pour limiter les problèmes de séparation et de purification.

3. La conception de synthèses moins dangereuses.

4. La conception de [...] molécules non toxiques

5. La recherche d'alternatives aux solvants polluants et aux auxiliaires de synthèse.

6. La limitation des dépenses énergétiques

7. L'utilisation de ressources renouvelables à la place des produits fossiles.

8. La réduction du nombre de dérivés en minimisant l'utilisation de groupes protecteurs ou auxiliaires.

9. L'utilisation des procédés catalytiques de préférence aux procédés stoechiométriques.

10. La conception des produits en vue de leur dégradation finale.

11. La mise au point des méthodologies d'analyses en temps réel pour prévenir la pollution,

12. Le développement d'une chimie prévenant accidents, explosions, et émissions de composés dangereux.

Source : D'après Anastas et Warner, 1998, p. 30.

Dans les faits, la Green Chemistry va ainsi être faite de priorités données à certains de ces 12 préceptes et de compromis entre ceux-ci. Les scientifiques vont donc pouvoir, par exemple, progresser de façon significative sur des principes comme l'économie d'atome ou la catalyse et l'économie d'énergie sans pour autant réaliser des produits ou des process non-toxiques. À cet endroit, on voit que si la chimie durable du végétal est bien dans la logique du développement d'une Green Chemistry ( $7^{\mathrm{e}}$ principe, figure 2 ), cette position ne va pas pour autant donner une vision convergente de la forme qu'elle devrait prendre puisqu'elle va, de fait, faire elle-même l'objet de compromis variables.

\section{L'hétérogénéité de la place de chimie du végétal dans la Green Chemistry}

La chimie du végétal ${ }^{7}$ apparaît dans les 12 principes au travers du $7^{\mathrm{e}}$ précepte qui préconise l'utilisation de ressources renouvelables. Selon les

7. Par « chimie du végétal » on entend ici toutes les valorisations de la biomasse reconnues par l'ADEME que ce soit en chimie (agromolécules et agromateriaux) ou en énergie (agrocombustibles et agrocarburants). 
communications de l'ACS Symposium sur la Green Chemistry des années 1990, cette préconisation est essentiellement liée à la recherche de diminutions des risques et des dangers dans la mesure où la biomasse constituerait un moyen de substituer des molécules synthétiques créées ex nihilo, et dont on connaît mal la toxicité, par des molécules présentant, a priori, des caractéristiques non toxiques et biodégradables.

Létude des réseaux nationaux de chimistes dédiés à la Green Chemistry montrent que la valorisation des ressources végétales s'inscrit dans la logique d'établissement de priorité et de compromis décrite précédemment. Certains de ces réseaux donnent bien la priorité au $7^{\mathrm{e}}$ principe et réalisent des compromis autour de celui-ci avec les autres principes. C'est le cas des réseaux de recherche Canadien, Brésilien, Espagnol et Japonais. Tandis que d'autres réseaux de recherche (États-Unis et Royaume-Uni) associent plutôt la valorisation de la biomasse avec d'autres principes sans pour autant y donner une priorité évidente. Cette même différence ressort dans les ouvrages de référence mondiaux sur le concept ${ }^{8}$. Certains numéros de l'ACS Symposium Serie traitent de Green Chemistry sans pour autant parler de biomasse ${ }^{9}$ alors que l'ouvrage de Paul Colonna, intitulé La Chimie Verte (2006), marque un véritable coup de force de l'INRA sur la notion de Green Chemistry dans la mesure où dans cette somme la Green Chemistry et la chimie du végétal se confondent complètement. L'étude de ces matériaux empiriques souligne donc que lorsqu'un compromis opéré au sein des 12 principes est fait en faveur de la biomasse, alors les solutions de couplage avec les autres principes de la Green Chemistry sont multiples et aucune voie de développement de la chimie verte du végétal ne se révèle dominante. Par exemple, certains scientifiques ont tenté de mettre en œuvre une chimie du végétal en l'associant à la question de la biodégradabilité ( $10^{\mathrm{e}}$ principe) pour les valorisations de l'amidon. D'autres ont plutôt privilégié l'association « biomasse » et « alternatives aux solvants polluants et aux auxiliaires de synthèse » ( $5^{\mathrm{e}}$ principe). In fine, si la mise en place d'un cadre conceptuel pour la chimie durable a permis de souligner le rôle important que la biomasse avait à jouer dans cette perspective, elle n'a pas pour autant défini une voie technologique dominante qui caractériserait la composante technico-scientifique du SSI C2V.

8. Les publications de l'ACS Symposium Series depuis 1998, Anastas, Warner, 1998 ; Clark, Macquarrie, 2002 ; Colonna, 2006.

9. e.g. les publications sur les liquides ioniques. 


\section{La chimie du végétal repositionnée au cœur de la chimie verte et le maintien de la diversité des voies technologiques}

\section{Un renversement de perspective qui pose la chimie du végétal comme élément central de la chimie durable}

De notre point de vue, si l'on revient au cas particulier de l'ouvrage « La chimie verte » de Colonna, la publication d'un tel ouvrage assimilant Green Chemistry / chimie du végétal révèle des déplacements dus à la montée en puissance des questions relatives à l'usage des ressources renouvelables en chimie depuis les années 2000. D'autres indicateurs confortent d'ailleurs cette interprétation. On peut citer, par exemple, les Presidential Green Chemistry Challenge donnant une place toujours plus importante aux travaux relatifs à ces ressources. Sur les 15 premiers prix (délivrés entre 1996 et 1998), deux seulement peuvent être rattachés à une chimie du végétal. Sur les trois dernières années, 11 prix sur 15 peuvent être directement rattachés à des produits biobasés. Il en est de même pour les revues, Green Chemistry ou Chemsuschem. Pour la première, fin 2011, sur 63 articles à paraître dans l'année 2012 publiés sur le site de la revue, 57 relèvent du 7ème principe, alors qu'aucun n'apparaissait dans le premier numéro de la revue. Pour Chemsuschem, les deux premiers numéros publiés en 2008 n'avaient aucun article référencé avec le mot-clé biomass, et aucun article comportant le terme renewable ressources n'était présent dans cette revue avant le $8{ }^{\text {ème }}$ numéro. Elle en compte aujourd'hui 103 sur le premier mot-clé et 88 sur le second, contre 41 articles référencés avec le mot clé Green Chemistry sur toute la période d'existence de la revue ${ }^{10}$. Aussi, peut-être plus qu'une simple assimilation Green Chemistry / chimie du végétal, nous pensons qu'aujourd'hui le mouvement doit-être être vu comme un renversement de perspective.

En se rappelant que la chimie durable est avant tout une approche holistique ayant pour objectif final la réalisation de la soutenabilité générale de la société grâce à la chimie ${ }^{11}$, ces mouvements montrent qu'au cours des années 2000, en faisant le choix de maintenir une chimie du carbone - c'est-à-dire une discipline pour laquelle il n'existe pas d'autres alternatives au carbone fossile que le carbone d'origine renouvelable -, la chimie durable se trouve à la croisée de chemins. L'inventaire des connaissances scientifiques et des savoir-faire (Colonna, 2006 ; Clark, Deswarte, 2008) nécessaires à la mise en

10. Consultation des revues 13 décembre 2011.

11. Certes, elle inclut, bien sûr, la green chemistry, mais elle renvoie avant tout à un objectif d'" équilibre entre croissance économique et développement, préservation de l'environnement, et promotion de la société (protection de la santé et qualité de la vie) »(Centi, Perathoner, 2009). 
œuvre de ce type de chimie abonde dans ce sens en indiquant un challenge scientifique et technique pouvant être résumé de la sorte : la chimie du végétal peut être plus «sale $»^{12}$ et plus difficile à mettre en œuvre ${ }^{13}$.

Les exemples abondent également dans les revues de littérature des scientifiques menées sur le végétal (Kamm, Kamm, 2004 ; Clark, Dewarte, 2008 ; Bozelle, Petersen, 2010 ; Sheldon, 2011 ; Gallezot, 2012) confirmant ce renversement de perspective majeur plaçant la ressource renouvelable au cœur du dispositif. Celui-ci oblige alors les acteurs à s'interroger sur la manière dont ils peuvent rendre cette chimie du végétal à la fois green and sustainable ${ }^{14}$. La réponse passe alors une réarticulation des 11 autres outils de chimie verte autour de la biomasse afin de servir la double préoccupation de la soutenabilité environnementale et économique via l'usage des ressources renouvelables (Figure 3) : «L'utilisation de matières premières renouvelables est le $7^{e}$ principe de la liste des principes de chimie verte, mais les processus de conversion de la biomasse ne doivent pas ignorer les 11 autres principes » (Gallezot, 2012). C'est à cet endroit que nous retrouverons alors notre question des voies technologiques de mise en ouvre de la C2V.

Figure 3 - Schéma des outils de la Green Chemistry recomposés autour du végétal pour aller vers une chimie doublement verte Garnier (2012)

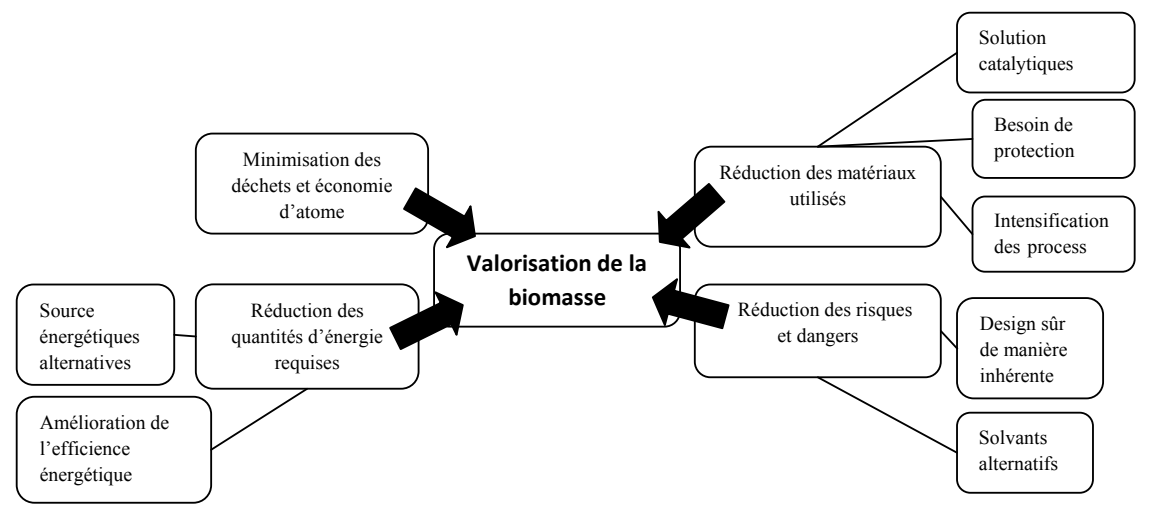

12. Compte tenu des impuretés, des coproduits et de la variabilité de la matière première.

13. Une partie importante des réactions de synthèse documentées aujourd'hui sur des biomasses sont fondées sur des réactions obtenant de faibles taux de rendement réactionnels à l'instar des réactions catalytiques que l'industrie pétrolière a énormément explorées pour optimiser ses process.

14. Expression d'un éditorial de Green Chemistry (Sheldon, 2008). 


\section{Les voies technologiques de la C2V définies par des préoccupations économiques, environnementales et par des héritages productifs}

Au travers des entretiens, revues de littérature ou études des projets de recherche, on peut percevoir à cet endroit que les scientifiques ont tendance à se spécialiser sur les espérances à la fois économiques et technologiques portées par les solutions techniques des différents principes de la Green Chemistry (nouveaux substrats, solvants, catalyseurs, etc.). Mais encore une fois, force est de constater qu'aucune voie dominante n'émerge. Cette absence est d'ailleurs marquée par la parution de nouveaux journaux spécialisés (comme Polymers from Renewable Ressources, 2009 ; Biofuels, Bioproducts and Biorefining, 2007).

En fait, les scientifiques explorent de façon très spécifique le domaine d'application qu'ils maîtrisent et les réactions originales qu'ils sont capables de mettre en forme. En parallèle, ils s'interrogent sur l'endroit où ces réactions, qui relèvent de la recherche fondamentale, pourraient être rattachées au tableau des différents débouchés des valorisations de la biomasse. Par exemple, le programme PIR Ingenotech rassemblant chimistes et économistes observe la mise en forme vers des usages industriels de travaux en recherche fondamentale. On retrouve donc bien derrière cette initiative l'idée que les scientifiques ne peuvent se cantonner au seul niveau de principes appliqués en laboratoire. Ils ont besoin d'inscrire la C2V dans un contexte plus large en considérant les domaines d'application de leurs innovations à la fois du point de vue environnemental et économique.

Par ailleurs, de notre point de vue, les multiples voies technologiques investies par les scientifiques sont aussi en grande partie dues à la présence d'« héritages productifs » (Nieddu et al., 2011 ; Garnier, 2012). Dans le cadre des travaux menés conjointement avec des scientifiques de l'INRA et du CNRS participants au projet APERC2V, nous avons constaté que chacune des stratégies de recherche et développement menées au niveau du fractionnement et du traitement du végétal relevait, en fait, de connaissances et de compétences bien maîtrisées dans d'autres domaines. Celles-ci sont réutilisées pour réaliser la transition de la chimie pétrosourcée à une chimie biosourcée. La synthèse de ces éléments fait alors surgir quatre « héritages productifs » (Bliard et al., 2011). Le premier (HP1) renvoie à une déconstruction thermique complète de la biomasse. Le deuxième (HP2) correspond à une dégradation enzymatique de composé de la biomasse. Le troisième renvoie à la valorisation des composés existant naturellement dans la biomasse après une extraction et des modifications par des procédés chimiques très limités. Enfin, le dernier correspond à l'extraction en l'état de composés organiques de taille importante et à leur fonctionnalisation. 
Dans la mesure où, d'un côté, un même produit fini peut être obtenu à partir de différentes matières premières et/ou différents process chimiques et que d'un autre côté on a un contexte de ressources limitées, la science chimique est une science qui se doit de limiter les voies qu'elle investit. La science chimique est donc une science " orientée ». Néanmoins après les travaux que nous avons engagés sur la composante académique de la $\mathrm{C} 2 \mathrm{~V}$ et que nous avons présentés dans cette partie, force est de constater qu'il n'y a pas, à ce niveau du SSI C2V, une convergence des voies scientifiques explorées qui mènerait au développement d'un nombre de voies technologiques limité. Néanmoins, compte tenu des enseignements de cette dernière sous-section, la diversité des voies envisagées n'apparaît pas non plus comme une diversité " anarchique " puisqu'elle peut être mise en ordre (figure 4).

Figure 4 - Une mise en ordre de la diversité des voies technologiques de la C2V

\begin{tabular}{|c|c|c|c|c|}
\hline $\begin{array}{l}\text { Principes } \\
\text { de chimie }\end{array}$ & $\begin{array}{c}\text { Logique } \\
\text { économique }\end{array}$ & \multicolumn{2}{|c|}{ Héritage productif } & $\begin{array}{l}\text { Logique environnemen- } \\
\text { tale dans une optique } \\
\text { Green Chemistry }\end{array}$ \\
\hline \multirow{3}{*}{$\begin{array}{l}\text { Fraction- } \\
\text { nement } \\
\text { déstruc- } \\
\text { turant }\end{array}$} & \multirow{3}{*}{$\begin{array}{l}\text { S'intégrer } \\
\text { dans la supply } \\
\text { chain de la } \\
\text { pétrochimie par } \\
\text { des innovations } \\
\text { « modulaires » } \\
\text { et en livrant les } \\
\text { intermédiaires } \\
\text { adéquats }\end{array}$} & \multirow{2}{*}{$\begin{array}{l}\text { Mobilisation } \\
\text { des procédés } \\
\text { thermochimiques } \\
\text { liés à la chimie } \\
\text { traditionnelle (HP1) }\end{array}$} & Gazéification & $\begin{array}{l}\text { Catalyse homogène } \\
\text { et hétérogène }\end{array}$ \\
\hline & & & Pyrolyse & $\begin{array}{l}\text { Rupture dans les } \\
\text { systèmes (plasma) }\end{array}$ \\
\hline & & \begin{tabular}{|l|} 
Hybridation de la \\
pétrochimie avec \\
des procédés \\
biochimiques liés \\
à l'agroalimentaire \\
(HP2) \\
\end{tabular} & $\begin{array}{l}\text { Procédés } \\
\text { fermentaires }\end{array}$ & $\begin{array}{l}\text { Catalyse enzymatique } \\
\text { ou microbienne }\end{array}$ \\
\hline \multirow[b]{3}{*}{$\begin{array}{l}\text { Fraction- } \\
\text { nement non } \\
\text { déstructurant } \\
\text { ou déstructu- } \\
\text { ration limitée }\end{array}$} & \multirow{3}{*}{$\begin{array}{l}\text { Utiliser des } \\
\text { supply chain } \\
\text { existantes dans } \\
\text { l'alimentaire et } \\
\text { la papeterie en } \\
\text { contournant les } \\
\text { fournisseurs } \\
\text { d'intermédiaires } \\
\text { chimiques } \\
\text { traditionnels }\end{array}$} & \multirow{3}{*}{$\begin{array}{l}\text { Mobilisation des } \\
\text { compétences de } \\
\text { l'agro-alimentaire } \\
\text { et de la papeterie } \\
\text { en matière de } \\
\text { complexité du } \\
\text { vivant et de } \\
\text { traitement non } \\
\text { déstructurant de la } \\
\text { matière première } \\
\text { (HP3 et HP4) }\end{array}$} & $\begin{array}{l}\text { Catalyse One } \\
\text { pot cascade }\end{array}$ & \multirow[b]{3}{*}{$\begin{array}{l}\text { Économie d'étapes et } \\
\text { donc d'énergie, process } \\
\text { en continu }\end{array}$} \\
\hline & & & Photochimie & \\
\hline & & & $\begin{array}{l}\text { Extrusion } \\
\text { réactive }\end{array}$ & \\
\hline
\end{tabular}




\section{LA DYNAMIQUE DE LA COMPOSANTE TECHNICO-SCIENTIFIQUE DU SYSTĖME SECTORIEL D'INNOVATION DE LA CHIMIE DOUBLEMENT VERTE AU NIVEAU INDUSTRIEL : LE CAS DES FILIĖRES DES REVÊTEMENTS DE SOL RÉSILIENTS}

Après avoir vu que, malgré le caractère de science orientée qui définit la science chimique, différentes voies scientifiques mettant en œuvre les préceptes d'une chimie doublement verte étaient maintenues, nous allons maintenant porter notre analyse sur l'aval du secteur. Nous allons traiter de filières particulières et étudier les stratégies d'innovation qui les animent. En se replaçant dans le contexte théorique, et donc en se référant au schéma en deux temps « exploration-génération de variété »/« sélection-exploitation » des voies technologiques, l'objectif de cette deuxième partie est de voir si les filières de la $\mathrm{C} 2 \mathrm{~V}$ passent bien par cette phase de réduction des voies technologiques investies (et donc des bases scientifiques mobilisées), ou, si au contraire, on a un maintien de la diversité. Les filières que nous avons retenues sont celles des revêtements de sol résilients dans la mesure où ceux-ci ont été pleinement confrontés aux enjeux de développement durable exposés en introduction. Nous allons donc dans cette seconde partie étudier les stratégies d'innovation des trois principaux acteurs du secteur des revêtements de sol résilients sur le marché de l'Europe de l'Ouest. Notre but sera de voir si, à ce niveau, il y émergence d'une voie technologique dominante basée sur des développements scientifiques particuliers et orientant le SSI C2V ou si la diversité des voies et des héritages productifs associés qui ont été présenté dans la partie1 se maintient.

\section{Le secteur des revêtements de sol résilients}

\section{Qu'est-ce qu'un revêtement de sol résilient?}

Les « revêtements de sol résilients » correspondent à l'ensemble des produits de revêtement de sol ayant la caractéristique de reprendre leur forme initiale malgré diverses compressions et manipulations lors de leurs processus de pose, d'utilisation et d'entretien. L'Institut Européen des producteurs de revêtements de sol résilients identifie huit familles de revêtements de sol résilients présentant des caractéristiques secondaires ${ }^{15}$ différentes en

15. En termes de confort, d'isolation phonique, de mode de mise en œuvre, de design, etc. 
fonction de leurs composants et de leurs procédés de production (ERFMI, 2005 $)^{16}$. Cinq d'entre elles : les PVC homogènes, les PVC hétérogènes, les PVC mousses, les PVC expansés, le PCV semi-flexibles, le PVC anti-glissement sont d'origine petrosourcée et, selon l'ERFMI, constituent ensemble le groupe de revêtement de sol dominant le marché aujourd'hui. Néanmoins, cette hégémonie du PVC se confronte à deux autres familles, le linoléum et les revêtements de sol en caoutchouc, qui sont élaborées à partir de ressources d'origine végétale.

Le linoléum résulte d'un procédé ancien renvoyant au HP4 et reposant sur l'extrusion et le séchage de la matière végétale. C'est un produit qui se rapproche du PVC au niveau de ses performances acoustiques et dans la mesure où il convient aux lieux très fréquentés. De même, au niveau du design, son utilisation est compatible avec la mise en œuvre de nuances de couleurs similaires aux produits PVC homogènes et expansés. Par contre, contrairement aux produits petrosourcés, l'utilisation du linoleum se voit limitée aux zones sèches.

In fine, on a donc un secteur d'activité des revêtements de sols résilients qui repose à la fois sur des filières de valorisation des ressources d'origines végétales et sur des filières de valorisation des ressources pétrolières, ces dernières étant parfois complémentaires et parfois concurrentes en termes de fonctionnalité.

\section{Les opérateurs du secteur en Europe de l'Ouest}

En cantonnant notre étude à l'Europe de l'Ouest ce sont trois opérateurs principaux qui nous sont apparus comme tenant le marché des revêtements de sol résilients : le groupe Tarkett, le groupe Forbo International et Gerflor. Si l'histoire de chacun est marquée par des trajectoires différentes: Tarkett vient de la valorisation du bois en parquet au $19^{\mathrm{e}}$ siècle et a évolué vers la production de revêtement de sols PVC après la Seconde Guerre mondiale, Forbo du linoleum au début des années 1920, et Gerflor du vinyle dans les années 1930 ; l'étude des gammes de produits qu'ils proposent nous permet de constater qu'aujourd'hui ils ont tous les trois investis à la fois le segment des produits provenant du PVC et celui des produits issus de la biomasse. Dans le détail, l'exploitation de ces deux segments par ces industriels présente en fait un paysage concurrentiel très différent selon que l'on soit du côté du PVC ou du linoléum. Le groupe Tarkett détient plus d'un tiers des parts de marché des produits PVC en Europe de l'Ouest tandis que les groupes Gerflor et

16. Dans la suite nous parlerons d'une voie « hybride». On notera que cette voie ne constitue pas, pour le moment, de famille référencée par l'ERFMI. 
Forbo en possèdent respectivement $10 \%$ et $5 \%$. Cette domination s'inverse sur le marché du linoléum sur lequel Forbo s'impose comme un des leaders européens, laissant loin derrière lui Tarkett alors que Gerflor ne joue qu'un rôle de distributeur (Entretiens, 2012 17 ).

\section{La prise en compte du végétal dans les stratégies d'innovation à partir de l'étude des couples " produits développés/rapport à l'environnement " sur le long terme}

L'arbitrage par les industriels entre la valorisation des ressources pétrolières et celle des ressources issues de la biomasse dans le secteur des revêtements de sols résilients n'a pas suivi une trajectoire linéaire. En fonction des évolutions du paysage dans lequel ils ont évolué, les industriels ont développé des produits spécifiques et, pour des raisons économiques et/ou environnementales ${ }^{18}$, ont accordé une place variable à la valorisation de la biomasse. Létude des stratégies des acteurs nous a permis de mettre en avant 1) deux périodes différentes reposant sur des couples spécifiques «produits/rapport à l'environnement» et 2) des stratégies diverses de mise en ouvre de la C2V dans la seconde période identifiée.

\section{Une première période caractérisée un rapport à l'environnement à géométrie variable en fonction de dynamiques économiques globales}

La première période identifiée se caractérise par un premier moment dans lequel les opérateurs du secteur se sont développés en fonction des ressources, matérielles et/ou immatérielles, disponibles à proximité de leurs sites de production. Par exemple, le groupe Gerflor implanté dans le Rhône s'est initialement positionné sur la production de revêtements de sol en vinyle dans une région spécialisée dans les métiers de la plasturgie. La description de ce premier moment nous apprends deux choses : 1) que les trois leaders actuels du marché possèdent des savoir-faire initiaux différents (le bois, le textile,

17. Informations recueillies lors d'entretiens auprès des industriels du secteur.

18. Les premières critiques ont porté sur la dangerosité de l'utilisation de matériaux tels que l'amiante, le cadmium, les solvants et la paraffine chlorée dans les process de production des produits en PVC dans les années 1970 (Schwartz, 2006 ; Tarkett, 2006). À la fin des années 1980, c'est un élément central de la composition du PCV qui a été contesté : le chlore (Schwartz, 2006). Deux autres problèmes ont ensuite été soulignés pour les produits PVC : la dangerosité des phtalates (Vynil, 2010), les émissions de composés organiques volatils (COV) (Jönsson, 1999 ; Commission Européenne, 2004). Ce problème des COV concerne en fait aussi le linoléum (Jönsson, 1999). Les autres enjeux environnementaux partagés par les deux produits sont : l'épuisement des ressources fossiles et les process d'entretien consommateurs de détergents, d'eau et d'énergie (ERFMI, 2005 ; Pluijmert et al., 2008). 
le PVC et le linoléum) et, 2) qu'à l'origine leur rapport à l'environnement dépendait, non pas de questions de pollution où sanitaires comme ce sera le cas dans la seconde période, mais qu'il était uniquement lié à l'exploitation des ressources disponibles localement.

La fin de la Seconde Guerre mondiale marque un basculement de stratégies et le début d'un deuxième moment. Si l'émergence de la société de consommation incite à cette époque les principaux industriels à étoffer leurs portefeuilles d'activité en faisant du PVC et du linoléum deux familles de produits incontournables (Potting, Blok, 1995 ; Schwartz, 2006) ; la conjecture du faible prix du pétrole, de la hausse du prix du bois et de la nécessité de reconstruire une Europe dévastée, forme un contexte plaçant tout de même les produits pétrosourcés devant les biosourcés. De cette manière, par exemple, Tarkett réorganise en 1947 son processus de production de manière à proposer de revêtements de sol prenant la forme de dalles de PVC (Tarkett, 2006 ; Schwartz, 2006). Dans ce second moment prospère, alors que la question de l'ancrage territoriale et de la proximité de la matière première est en recul, et que les questions de développement durable (sécurité sanitaire et innocuité) ne sont pas encore prégnantes, le rapport entre les produits développés et la question environnementale apparait très distendu. L'avènement de la problématique environnementale ${ }^{19}$ menant à la mise en œuvre d'une stratégie collective pour aller vers une chimie durable va faire basculer les acteurs du secteur des revêtements de sols résilients dans une seconde période. Dans celle-ci, le rapport à l'environnement va présenter deux caractéristiques : l'importance accordée aux questions relatives au développement durable et la stabilité de cette importance dans le temps.

\section{Une mobilisation collective pour aller vers une chimie durable}

Le début des années 1990 marque donc l'émergence d'une seconde période pour la prise en compte des questions de développement durable par le secteur des revêtements de sols résilients en Europe de l'Ouest. Au cours de celle-ci, les acteurs industriels et les disciplines scientifiques associées à ce secteur se mobilisent pour apporter ensemble une réponse à la problématique environnementale. Par des actions collectives pilotées par les industriels eux-mêmes, on voit alors se cristalliser les fondements des principes d'une chimie durable pour les revêtements de sols résilients autour d'une recherche 1) d'une réduction des déchets (correspondant en fait au $1^{\mathrm{e}}$ et $10^{\mathrm{e}}$ principe de la Green Chemistry), 2) d'une réduction de l'énergie (6 $6^{\mathrm{e}}$ principe) et 3 ) d'une substitution de substances dangereuses ( $4^{\mathrm{e}}$ et $10^{\mathrm{e}}$ principe).

19. Voir note 27. 
Cette volonté d'agir ensemble se formalise en plusieurs étapes (Schwarz, 2006). Au début des années 1990 les industriels se rassemblent dans une structure unique : l'European PVC Flooring Manufacturers. Ensuite, quelques années plus tard, est mis en place de l'ERFMI afin d'accompagner les industriels dans leurs projets de développement. Enfin, ces actions collectives ont permis plus récemment au secteur des revêtements de sol résilients d'intégrer une communauté plus large : celle des industriels du plastique et ainsi de participer à des grands projets européens tels que le programme de recherche sur le recyclage des revêtements de sol (Vynil 2010).

L'ensemble de ces collaborations ont permis aux industries du secteur des revêtements de sols de minimiser la quantité de déchets et d'énergie en développant et optimisant, par exemple, des échanges de flux dans une logique d'économie circulaire, ou bien en s'appuyant sur des centres de recyclage au plus proche des sites de production. Il leur a également permis de développer des solutions alternatives aux phtalates et à l'émission de composés organiques volatils.

Au travers de ces actions collectives, c'est une vision commune de ce que peut être une chimie durable du secteur des revêtements de sols résilients partagée par les industriels et une convergence des efforts réalisés en ce sens qui apparaît. Pour autant, pour répondre à notre problématique initiale, il nous faut encore déterminer quelle est la place du végétal dans cette stratégie et voir comment la conception du linoléum et des produits d'origine PVC est influencée par les objectifs que s'est donnés le secteur.

\section{Des voies de développement différentes des revêtements de sols résilients relevant de la C2V}

De par son origine végétale, la recherche d'un niveau de durabilité supérieur pour le linoleum, positionne d'emblée le produit sur une stratégie " chimie doublement verte ». Compte tenu des objectifs globaux du secteur des revêtements de sol résilients en termes de développement durable, les efforts faits dans cette filière ont porté principalement sur l'association du $7^{\mathrm{e}}$ principe de la Green Chemistry avec le $1^{\mathrm{e}}$ (éviter la production de résidus). La complexité du produit et la faiblesse d'un dispositif de collecte rendent difficile son recyclage, les efforts pour la diminution des déchets liés à la production du linoleum sont passés par deux autres canaux. D'abord la valorisation de la plante entière. Ensuite, Tarkett et Forbo se sont engagés, dans une logique cradle to cradle, en bénéficiant de sa compostabilité et de son fort potentiel d'incinération du produit. De ce point de vue, le $7^{\mathrm{e}}$ principe de la Green Chemistry se retrouve alors par un effet rebond également associé au $6^{\mathrm{e}}$ principe (économie d'énergie). 
La réglementation REACH de 2007 a conduit les producteurs de revêtements de sol résilients a rattaché les filières PVC à la C2V en développant une voie spécifique. Les industriels ont hybridé ce PVC avec des molécules plateformes d'origine végétale pour lier les avantages du PVC (la recyclabilité) avec ceux de la biomasse (la renouvelabilité de la matière première) tout en réduisant les risques liés aux phtalates. Depuis peu, Tarkett développe ainsi l'IQ natural, un produit issu de l'huile de ricin et recyclable (Tarkett, 2012). De même Forbo s'est investi dans la résine biosourcée (ARD, 2010) et Gerflor s'est engagé dans l'utilisation de molécules biosourcées (Polysorb de Roquette) s'intégrant dans des produits PVC recyclables (Convers, Thumerel, 2011 ; Formule verte, 2011). Ces molécules de substitution ne sont que des polymères clefs résultant d'un procédé thermochimique ou biochimique. Dès lors, ces nouveaux produits hybrides apparaissent comme une stratégie mixte alliant Green Chemistry et chimie doublement verte, i.e. comme un mélange entre les principes de la Green Chemistry et les héritages productifs 1 et 2 de la $\mathrm{C} 2 \mathrm{~V}$.

\section{Une voie de développement des revêtements de sol résilients dans une logique de Green Chemistry qui occulte la question de la valorisation du végétal}

À côté de cette nouvelle voie technologique reposant sur l'hybridation du pétrosourcé et du biosourcé, toujours dans l'optique de diminuer les résidus, les filières PVC ont aussi investi une autre voie d'hybridation : l'alliance du PVC pur et de la matière mise à disposition lorsque ces produits atteignent leur fin de vie. Dans ce scénario, les industriels restent donc sur des produits $100 \%$ petrosourcés. La capacité de valorisation de ces derniers dépend principalement de leurs complexités (les processus de déconstruction sont plus ou moins aisés selon les familles de produit) (ERFMI, 2005). En 2004, 7,5 \% de matières recyclées étaient incorporées dans un nouveau produit, et par la même contribuaient à une réduction de l'usage des ressources d'origine fossile et de l'énergie (ERFMI, 2005). Aujourd'hui, Gerflor proposent de nouvelles gammes de produits dans lesquelles se sont $32 \%, 53 \%, 55 \%$ et $100 \%$ de produits en fin de vie qui sont réinjectés. Aujourd'hui de nouvelles voies technologiques basées sur des processus thermochimiques de fonte et de refonte de la matière sont donc développées et permettent de rattacher les filières PVC à la chimie durable sans en passer par de la chimie doublement verte.

In fine, cette étude des stratégies développées aujourd'hui montre que la recherche de durabilité dans ce secteur se traduit au travers de la coexistence de plusieurs voies (Figure 5). Il y a d'un côté la mise en œuvre de voie technologique relevant de la Green Chemistry (i.e. pas de priorité sur le végétal) et de l'autre des voies relevant de la C2V (végétal et hybridation). 
Figure 5 - Diversité dans l'adoption de la CV et de la C2V au sein des produits PVC et linoléums du secteur des revêtements de sol résilients (réalisé par nos soins)

\begin{tabular}{|c|c|c|c|c|c|c|c|}
\hline \multirow{2}{*}{$\begin{array}{l}\begin{array}{l}\text { Identité } \\
\text { du produit }\end{array} \\
\text { Composition }\end{array}$} & \multicolumn{4}{|c|}{ Biosourcés (Linoleum) } & \multirow{2}{*}{\begin{tabular}{|c|}
$\begin{array}{c}\text { Hybrides } \\
\text { [PVC + } \\
\text { biosourcé] }\end{array}$ \\
Molécules \\
Plateformes
\end{tabular}} & \multicolumn{2}{|c|}{$\begin{array}{l}\text { Pétrosourcés } \\
\text { (PVC) }\end{array}$} \\
\hline & $\begin{array}{c}\text { Colophane } \\
\text { naturelle }\end{array}$ & Fibres & Bois & $\begin{array}{l}\text { Molécules } \\
\text { oléagineuses }\end{array}$ & & $\begin{array}{l}\text { Matières } \\
\text { plastiques } \\
\text { recyclées }\end{array}$ & $\begin{array}{c}\text { Matières } \\
\text { plastiques } \\
\text { pures }\end{array}$ \\
\hline Fonctionnalités & \multicolumn{7}{|c|}{ Traitement de surface - similarité } \\
\hline $\begin{array}{l}\text { Héritage } \\
\text { productif }\end{array}$ & \multicolumn{4}{|c|}{ Héritage Productif 4} & $\begin{array}{l}\text { Héritage } \\
\text { Productif } \\
1 \text { et } 2\end{array}$ & \multicolumn{2}{|c|}{$\varnothing$} \\
\hline Stratégie & \multicolumn{4}{|l|}{ 2CV } & $\begin{array}{l}\text { Stratégie } \\
\text { mixte } \\
{[\mathrm{C} 2 \mathrm{~V}+\mathrm{GC}]}\end{array}$ & \multicolumn{2}{|c|}{ Green Chemistry } \\
\hline Acteurs & \multicolumn{4}{|c|}{ Tarkett, Forbo International } & $\begin{array}{l}\text { Tarkett, } \\
\text { Forbo } \\
\text { International } \\
\text { Gerflor }\end{array}$ & \multicolumn{2}{|c|}{$\begin{array}{l}\text { Tarkett, Forbo } \\
\text { Flooring System, } \\
\text { Gerflor }\end{array}$} \\
\hline
\end{tabular}

\section{CONCLUSION}

L'objectif de ce papier était de savoir s'il y avait une convergence ou une divergence dans la dynamique d'évolution de la composante technico-scientifique du Système Sectoriel d'Innovation de la chimie doublement verte afin de saisir si ce dernier s'orientait vers une voie technologique dominante unique ou vers une diversité de voies maintenue dans le temps. Nos investigations nous ont menés à la conclusion qu'il y avait un maintien durable ${ }^{20}$ de la diversité des voies technologiques développées pour ce secteur. Si ce résultat, qui ne propose pas un dénouement unique, peut sembler plus difficile à défendre que d'autres résultats de recherche, c'est précisément cette caractéristique qui fonde tout son intérêt. Ce dernier suggère qu'on peut avoir un nouveau modèle avec des régimes technico-économiques dans lesquels on a un maintien de la variété des solutions. Pour être plus précis notre résultat nous fait en effet revenir sur le modèle canonique de la perspective multi-niveaux qui est implicitement construite autour du passage d'un régime technico-économique dominant fondé sur un artefact unique à un autre régime technico-économique dominant fondé sur un autre artefact unique. Dans notre cas le pétrole et sa raffinerie remplacé par la biomasse et la bioraffinerie. Finalement, il incite à enrichir ce modèle canonique par une représentation prenant en compte la possibilité d'un nouveau régime

20. Au sens « qui dure dans le temps». 
technico-économique d'une autre nature : un régime dont la caractéristique principale serait la nécessité du maintien d'une diversité des artefacts.

\section{BIBLIOGRAPHIE}

ANASTAS, P., WARNER, J. (1998), Green Chemistry - Theory and Practice, Oxford, Oxford University Press.

BLIARD, C. et al. (2011), Communities and creation of knowledge as common goods in doubly green chemistry, $3^{\text {rd }}$ International conference on biodegradable and Bio-based polymers, Biopol 2011, Strasbourg, 29-30 août.

BOZELL, J., PETERSEN, G. (2010), Technology development for the production of Biobased products from biorefinery carbohydrates - the US Department of Energy's Top 10 revisited", Green Chemistry, 12, 539-554.

CARSON, R. (1962), Silent Spring, Houghton Mifflin, 40 th edition.

CENTI, G., PERATHONER, S. (2009), in F. Cavani et al. (eds), Sustainable Industrial Processes, WILEY-VCH Verlag GmbH \& Co. KGaA, Weinheim enti et Perathoner.

CLARK, J., MACQUARRIE, D. (2002), Handbook of Green Chemistry and Technology, Blackwell Science.

CLARK, J. (1998a), From brown earth to green chemistry, Science in Parliament, 55, 10.

CLARK, J. (1998b), The greening of chemistry, Chemistry in Britain, 43-45.

CLARK, J. (1999c), Green Chemistry: challenges and opportunities, Green Chemistry, 1, 1-8.

CLARK, J., DESWARTE, G. (2008), Introduction to Chemical from biomass, Wiley.

COLONNA, P. (ed.) (2006), La chimie verte, Paris, Lavoisier, Tec \& Doc.

CONVERS, P., THUMEREL (2011), New flooring with 100\% biobased plasticizers. Disponible sur :

http://www.plantbasedchemistry.com/medias/File/ACDV\%2011\%20CP\%20Plant\%20 Based\%20Chemistry\%20Juillet\%20V2.pdf.

ENTREVUES (2012), Interviews with managers, chimists and engeneers in the sector of resilient floorings - our own elaboration, survey during 2011 \& 2012.

ERFMI (2011), News \& Information. European Resilient Flooring Manufacturer's Institute. Available, http://www.erfmi.com/news.php.

GALLEZOT, P. (2012), Critical Review: Conversion of biomass to selected chemical products, Chem. Soc. Rev.

FORMULE VERTE (2011), Revêtement de sol sans phtalate, Formule verte (5).

GARNIER, E. (2012), Une approche socio-économique de l'orientation des recherche en chimie doublement verte, thèse de doctorat, URCA.

GEELS, F. (2012), Technological transitions as evolutionary reconfiguration processes: A multi-level perspective and a case-study, Research Policy, 31(8-9), 1257-1274.

GEELS, F. (2005), Technological transitions and system innovations: a co-evolutionary and socio-technical analysis, Edward Elgar Publishing.

GEELS, F. (2005), Processes and patterns in transitions and system innovations: Refining the co-evolutionary multi-level perspective, Technol. Forecast. Soc. Change, 72, 681-696. 
GEELS, F. (2010), Ontologies, socio-technical transitions (to sustainability), and the multi-level perspective. Res. Policy, 39, 495-510.

GEELS, F. (2011), The multi-level perspective on sustainability transitions: Responses to seven criticisms, Environ. Innov. Soc. Transitions, 1, 24-40.

GRIN, J. et al. (ed.) (2010), Transitions to Sustainable Development: New directions, The Study of Long Term Transformative Change, London, Routledge.

HOLDER, R. (2002), Chemistry and the environment, in Clark, Macquarrie (eds), Handbook of Green Chemistry and Technology, Blackwell Science, 28-55.

JONSSON, A. (1999), Including the Use Phase in LCA of Floor Coverings, The International Journal of Life Cycle Assessment, 4(6), 321-328.

KAMM, B., KAMM, M. (2004), Biorfinery systems, Chem. Biochem. Eng., 13, 1-6.

LINTHORST, J. (2010), An overview: origins and development of green chemistry, Foundations of Chemistry, 12(1), 55-68.

MALERBA, F. (2002), Sectoral systems of innovation and production, Research Policy, 31, 247-264.

NILL (2003), Windows of sustainability opportunities - Determinant of techno-economic Time windows and conditions under wich encironmental innovation policy can utilise them, Paper for DRUID PhD Winter conference January 16th -18th, Aalborg, 20p.

NIEDDU, M. et al. (2010), L'émergence d'une chimie doublement verte, une approche en termes de démarche narrative, Revue d'Economie Industrielle, 132, 53-84.

NIEDDU M. et al. (2011), Towards sustained diversity in the new socio-technical regime of biorefinery?, Working paper, Lund, 20-21 june.

NILL, KEMP, R. (2009), Evolutionary approaches for sustainable innovation policies: From niche to paradigm?, Research Policy, 38(4), 668-680.

POTTING, J., BLOK, K. (1995), Life-cycle assessment of four types of floor covering, Journal of Cleaner Production, 3(4), 201-213.

PLUIJMERT, T. et al. (2008), Industry approach to life cycle assessment, Plastics, Rubber and Composites, 37(9-10), 406-410.

SARRADE, V. (2008), Quelles sont les ressources de la chimie verte?, EDP sciences.

SCHWARTZ, B. (2006), Environmental strategies as automorphic patterns of behavior, Business Strategy and the Environment, 18, 192-206.

SHELDON (2011), Utilisation of biomass for sustainable fuels and chemicals: Molecules, methods and metrics, Catalysis Today, 49.

TARKETT (2012), Déclaration du produit iQ Natural - PVC Homogène compacts, Available at: http://iqnatural.tarkett.fr/pdf/iqnatural_fr.pdf.

TARKETT (2006), Environmental statement - Ronneby - 2006, Ronneby, Suède. Available at: http://www.tarkett-commercial.com/download/fr-FR-3/Brochure-RonnebyEnvironmental-EN.pdf.

The European PVC Industry's Sustainable Development Programme, 2011, Vinyl 2010 - 10 years - reporting on the activities of the years 2010 and summarising the key milestones of the past 10 years. 